\title{
Metformin promotes autophagy and apoptosis in esophageal squamous cell carcinoma by downregulating Stat3 signaling
}

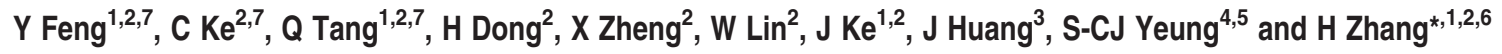

The antidiabetic drug metformin exerts chemopreventive and antineoplastic effects in many types of malignancies. However, the mechanisms responsible for metformin actions appear diverse and may differ in different types of cancer. Understanding the molecular and cellular mechanisms specific for different cancers is important to optimize strategy for metformin treatment in different cancer types. Here, we investigate the in vitro and in vivo effects of metformin on esophageal squamous cell carcinoma (ESCC) cells. Metformin selectively inhibited cell growth in ESCC tumor cells but not immortalized noncancerous esophageal epithelial cells. In addition to apoptosis, metformin triggered autophagy. Pharmacological or genetic inhibition of autophagy sensitized ESCC cells to metformin-induced apoptotic cell death. Mechanistically, signal transducer and activator of transcription 3 (Stat3) and its downstream target $\mathrm{Bcl}-2$ was inactivated by metformin treatment. Accordingly, small interfering RNA (siRNA)-mediated Stat3 knockdown enhanced metformin-induced autophagy and apoptosis, and concomitantly enhanced the inhibitory effect of metformin on cell viability. Similarly, the Bcl-2 proto-oncogene, an inhibitor of both apoptosis and autophagy, was repressed by metformin. Ectopic expression of Bcl-2 protected cells from metformin-mediated autophagy and apoptosis. In vivo, metformin downregulated Stat3 activity and Bcl-2 expression, induced apoptosis and autophagy, and inhibited tumor growth. Together, inactivation of Stat3-Bcl-2 pathway contributes to metformin-induced growth inhibition of ESCC by facilitating crosstalk between apoptosis and autophagy.

Cell Death and Disease (2014) 5, e1088; doi:10.1038/cddis.2014.59; published online 27 February 2014

Subject Category: Cancer

Because the antidiabetic drug metformin is associated with reduced risk of and improved prognosis of many cancers in type II diabetes patients ${ }^{1,2}$ and has an excellent safety record in diabetic patients, the clinical evaluation of metformin for its chemopreventive and antineoplastic effects has bypassed the traditional phase I assessment and has directly moved forward to phase II and phase III trials in several cancers. ${ }^{3,4}$ A growing body of epidemiological investigations and preclinical studies has implicated diverse mechanisms underlying the antitumor action of metformin, with different mechanisms playing important roles in different tissues and in different cancer types. ${ }^{1,2,4}$

Esophageal squamous cell carcinoma (ESCC) is the third most common malignancy of the digestive tracts and the sixth leading cause of cancer death in the world. ${ }^{5-8}$ Currently, there are no effective chemopreventive and therapeutic strategies for this lethal disease. However, the effects of metformin on ESCC have not been adequately studied and, in particular, the mechanisms by which metformin exerts its antitumor effects in ESCC have not been previously investigated. ESCC is an inflammatory malignancy in which inflammatory signaling pathways are aberrant. ${ }^{9-11}$ The transcription factor signal transducer and activator of transcription 3 (Stat3) is a point of convergence for numerous oncogenic and inflammatory signaling pathways, including cytokines, growth factors and oncogenes. ${ }^{12-14}$ Stat3 is a key player in inflammation-driven tumorigenesis, particularly ESCC, where its aberrant continuous activation is a signature of the disease. ${ }^{11-14}$ Moreover, Stat3 participates in cell growth and survival through regulating cell proliferation and apoptosis. ${ }^{15,16}$ Stat3 has emerged as a promising molecular target for cancer therapy. ${ }^{13}$ However, it is unknown whether the antineoplastic effects

\footnotetext{
${ }^{1}$ Department of Integrative Oncology, Affiliated Cancer Hospital of Shantou University Medical College, Shantou, China; ${ }^{2}$ Cancer Research Center, Shantou University Medical College, Shantou, China; ${ }^{3}$ Guangdong Provincial Key Laboratory of Infectious Diseases and Molecular Immunopathology, Shantou University Medical College, Shantou, China; ${ }^{4}$ Department of Endocrine Neoplasia and Hormonal Disorders, University of Texas MD Anderson Cancer Center, Houston, TX, USA; ${ }^{5}$ Department of Emergency Medicine, University of Texas MD Anderson Cancer Center, Houston, TX, USA and ${ }^{6}$ Tumor Tissue Bank, Affiliated Cancer Hospital of Shantou University Medical College, Shantou, China

${ }^{*}$ Corresponding author: H Zhang, Cancer Research Center, Shantou University Medical College, 22 Xinling Road, Shantou, 515041, China. Tel: +86 754 88900406; Fax: +86 754 88900406; E-mail: haozhang@stu.edu.cn

${ }^{7}$ These authors contributed equally to this work.

Keywords: metformin; esophageal squamous cell carcinoma; autophagy; apoptosis; Stat3; Bcl-2

Abbreviations: ESCC, esophageal squamous cell carcinoma; MTT, 3-(4,5-dimethylthiazol-2-yl)-2,5-diphenyltetrazolium bromide; DAPI, 4',6-diamidino-2phenylindole; MDC, monodansylcadaverine; 3-MA, 3-methyladenine; CQ, chloroquine; LC3, microtubule-associated protein light chain 3; siRNA, small interfering RNA; ATG, autophagy-related gene; AO, acridine orange; Stat3, signal transducer and activator of transcription 3; AMPK, AMP-activated protein kinase; mTOR, mammalian target of rapamycin; TUNEL, terminal deoxynucleotidyl transferase-mediated nick end labeling; IHC, immunohistochemistry; TEM, transmission electron microscopy

Received 13.9.13; revised 31.12.13; accepted 22.1.14; Edited by GM Fimia
} 
of metformin occur through inflammation-associated mechanisms in ESCC cells.

On the other hand, most published studies on the antitumor actions of metformin have focused on inhibition of proliferation and induction of apoptosis. ${ }^{17-19}$ Metformin has also been reported to cause autophagy, ${ }^{20}$ suggesting involvement of metformin in coordinating complex interaction between cell survival and growth. Understanding the interplay between apoptosis and autophagy induced by metformin may identify new targets for cancer therapy, and improve the therapeutic efficiency. Currently, there are no data about the effects of metformin on autophagy, and the interplay between autophagy and apoptosis in ESCC cells.

In this study, we investigated the in vitro and in vivo effects of metformin on human ESCC. In particular, we examined the role of Stat3 signaling in the interaction between apoptosis and autophagy mediated by metformin.

\section{Results}

Metformin selectively inhibited growth of human ESCC cells. To investigate the effect of metformin on growth of human ESCC cells, we used the EC109 and EC9706 human esophageal squamous cell carcinoma tumor cell lines, as well as the immortalized, noncancerous NE3 esophageal epithelial cell line. Metformin, in a concentration range of 1-20 mM, decreased cell viability of both EC109 and EC9706 cells over 24,48 and $72 \mathrm{~h}$ of continuous exposure, as assessed by MTT (3-(4,5-dimethylthiazol-2-yl)-2,5-diphenyltetrazolium bromide) assay (Figures 1a and b), but only marginally reduced the viability of NE3 cells (Figure 1c). In addition, in colony formation assays, metformin decreased colony formation compared with control (Figure 1d). Therefore, metformin inhibited ESCC cell growth.

\footnotetext{
Metformin induced ESCC apoptotic cell death and inhibited cell proliferation. To determine whether metformin causes cell death by apoptosis, ESCC cells were analyzed by flow cytometry following Annexin V-FITC and propidium iodide $(\mathrm{PI})$ dual labeling. As shown in Figure 2a, metformin $(5 \mathrm{mM})$ treatment for 48 and $72 \mathrm{~h}$ and for 7 days increased the number of apoptotic cells compared with their respective controls. As apoptosis is often associated with the collapse of mitochondrial membrane potential (MMP), ${ }^{5}$ the ability of metformin to depolarize the mitochondrial membrane was investigated by JC-1 staining. When mitochondria are polarized, $\mathrm{JC}-1$ is concentrated in the mitochondria to form aggregates that emit a red fluorescence. When mitochondria are depolarized, JC-1 cannot be concentrated in the mitochondria and continues to exist in a monomeric form that emits a green fluorescence. Consistent with prior reports, the red-to-green fluorescence ratio was found to decrease when cells were treated with metformin for $48 \mathrm{~h}$ (Figure 2b), indicating that metformin causes depolarization of the mitochondrial membrane.

TUNEL (terminal deoxynucleotidyl transferase-mediated nick end labeling) staining and western blot assay were performed to confirm induction of apoptosis. Treatment with metformin increased TUNEL-positive cells in a dose-
}

dependent manner (Figure 2c). As key executors of cell apoptosis, both caspase-cleaved PARP and the ratio of Bax to $\mathrm{Bcl}-2$ proteins increased in ESCC cells when treated with metformin for $24 \mathrm{~h}$, or with $10 \mathrm{mM}$ metformin for indicated time points (Figure 2d). Given that very strong inhibition of cell growth by metformin was especially prominent after 7 days of exposure (Figure 1a), additional cellular events may occur. Metformin has been shown to affect the proliferation of several human cancer types. As expected, cellular proliferation represented by phospho-Histone $\mathrm{H3}(\mathrm{pH} 3)$ immunofluorescence was decreased, particularly on day 7 after metformin addition (Figure 2e). Together, these results demonstrate that apoptosis, as well as cell proliferation, are involved in the response of ESCC to metformin treatment.

Inhibition of metformin-induced autophagy sensitized ESCC cells to metformin-induced apoptotic cell death. Metformin can trigger autophagy. ${ }^{20}$ We therefore examined whether metformin induced autophagy by morphological and biochemical identifications. The increased acidic vesicular organelles (AVOs) are correlated with increased autophagosomes indicating the formation of autophagolysosomes. To analyze the machinery involved in the autophagic process, acridine orange $(\mathrm{AO})$ staining and monodansylcadaverine (MDC) staining were employed to detect the AVO formation. AO-label and MDC-label cells were visualized using a fluorescent microscope after $20 \mathrm{mM}$ metformin treatment at particularly $48 \mathrm{~h}$ time point (Figure $3 \mathrm{a}$, upper and middle). Metformin-induced AVO formation was quantified by flow cytometry after staining the cells with $\mathrm{AO}$, demonstrating a dose-dependent increased percentage of AVOs from 0.99 to $25.95 \%$ (Figure 3b).

Microtubule-associated protein light chain 3 (LC3) is a specific marker for autophagy initiation. EC109 cells were transfected with GFP-LC3 cDNA and were detected using a fluorescent microscope. Metformin-treated cells showed a punctate pattern of GFP-LC3 fluorescence, representing recruitment of LC3-II to autophagosomes and the formation of autophagic vacuoles, whereas the vehicle-treated control cells exhibited diffuse LC3-associated green fluorescence (Figure 3a, low). In agreement with the results of formation of GFP-LC3-labeled vacuoles by fluorescent microscopy, conversion of the cytoplasmic form of LC3 (LC3-I, $18 \mathrm{kDa})$ to the pre-autophagosomal and autophagosomal membrane-bound form of LC3 (LC3-II, $16 \mathrm{kDa}$ ) was biochemically demonstrated by western blot in a dose-dependent manner (Figure 3c). Moreover, induction of autophagy was identified by two wellestablished measurements of autophagy, that is, enhancement of Beclin-1, a component of the class III phosphatidylinositol 3-kinase complex essential for autophagosome formation, ${ }^{21}$ and degradation of p62, a protein facilitating autophagic degradation of ubiquitinated protein aggregation ${ }^{22}$ (Figure 3c).

Finally, autophagy was confirmed by transmission electron microscopy (TEM). EC109 cells treated with metformin for $48 \mathrm{~h}$ showed typical autophagic vacuoles, including accumulation of numerous autophagic vesicles with a distinct double membrane, compared with no or few autophagic vacuoles in control cells (Figure 3d). Taken together, our results indicate that metformin treatment induced autophagy in human ESCC cells. 

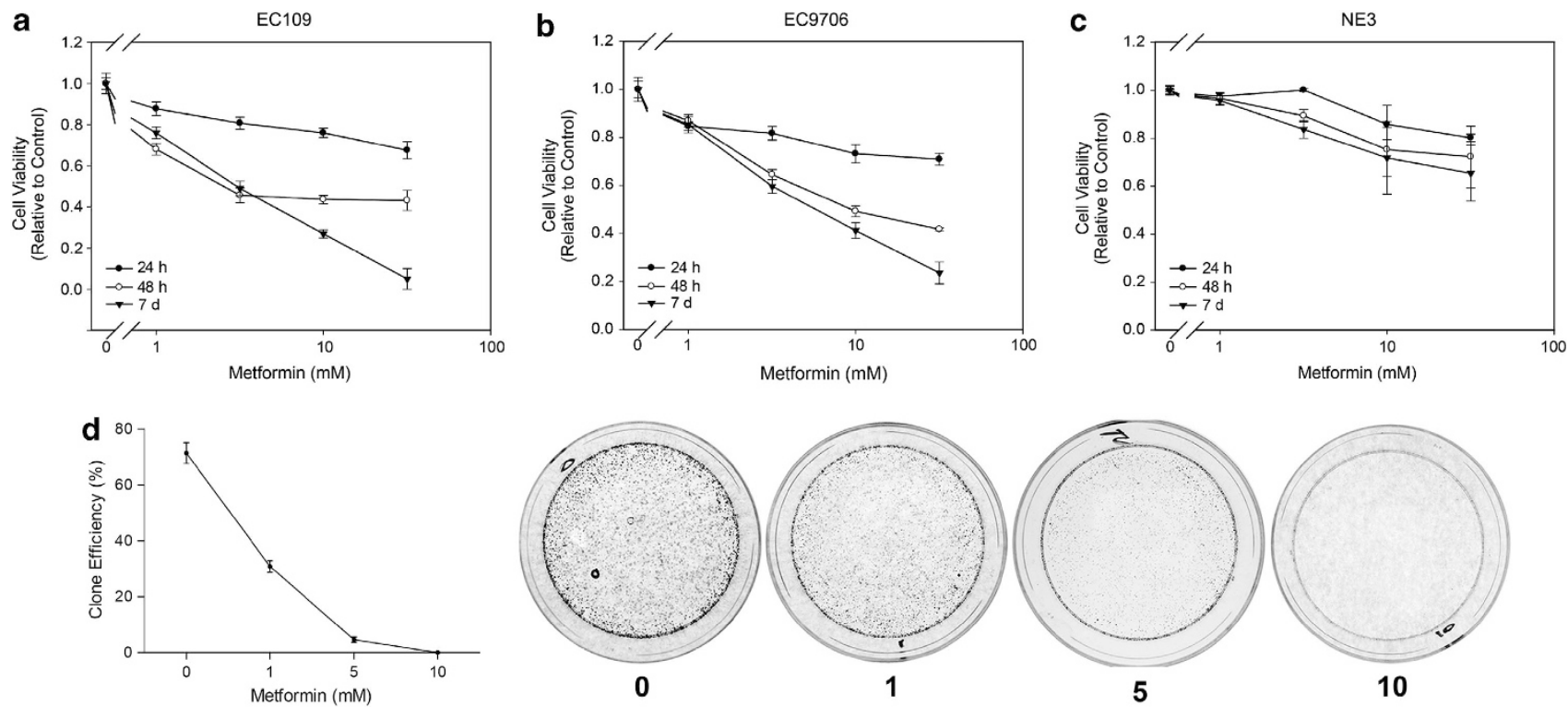

Figure 1 Metformin selectively inhibits ESCC cell growth. EC109 (a), EC9706 (b) and NE3 (c) cells were treated with metformin at the indicated concentration for 24 and $48 \mathrm{~h}$ and for 7 days. Cell viability, measured by MTT, was presented as the means \pm SD from three separate experiments. (d) Colony formation of ESCC cells was decreased in a dose-dependent manner by metformin treatment. Experiments were performed in triplicate

Autophagy is able to either suppress or promote tumor cell growth in different cellular contexts. ${ }^{23-26}$ Given that manipulation of autophagy may improve the efficacy of anticancer therapeutics, ${ }^{5,20}$ we were eager to determine whether the metformin-elicited autophagy in ESCC favored cell survival or cell death. We used two autophagy inhibitors: 3-methyladenine (3-MA), a phosphoinositide 3-kinase inhibitor that exerts its autophagy-inhibiting effect before the formation of autophagosomes, and chloroquine (CQ) that serves as a cancerspecific chemosensitizer partly dependent on its ability to inhibit autophagy. ${ }^{5,20}$ Pretreatment of cells with 3-MA or CQ decreased the number of viable metformin-treated cells, as assayed by MTT (Figure 4a). TUNEL staining of metformintreated cells was markedly enhanced in the presence of 3-MA (Figure 4b). Furthermore, pretreatment of cells with 3-MA or $\mathrm{CQ}$ enhanced the metformin-induced collapse of mitochondrial membrane potential (Figure $4 \mathrm{c}$ and Supplementary Figure 1). Pretreatment with 3-MA obviously decreased LC3II and Beclin-1, and increased Bax and cleaved PARP expression, indicating an elevated apoptosis process compared with metformin treatment alone (Figure 4d).

Pharmacological inhibitors of autophagy may exhibit autophagy-independent actions. ${ }^{27}$ Therefore, to corroborate the cytoprotective action of autophagy, the effects of metformin were examined in cells in which Beclin-1 and autophagy-related gene 5 (Atg5), two critical autophagic regulators, ${ }^{21,28}$ were downregulated by small interfering RNA (siRNA). EC109 cells transfected with Beclin-1 siRNA or Atg5 SiRNA showed a reduced level of LC3-II accumulation after metformin treatment compared with a scrambled siRNA control, indicating the involvement of Beclin-1 and Atg5 to metformin-mediated autophagy in ESCC (Figure 4e). In agreement with the data derived from pharmacological inhibitors, knockdown of Beclin-1 or Atg 5 by siRNA enhanced cleaved PARP, as assayed by western blot analysis
(Figure 4e), indicating that autophagy is cytoprotective for metformin-induced apoptotic cell death. In summary, metformin triggers autophagy in ESCC, and apoptosis is enhanced by inhibition of autophagy, suggesting that autophagy is a protective mechanism for ESCC cells in the context of metformin-induced apoptotic cell death.

Metformin inhibited Stat3/Bcl-2 pathway in an AMPKdependent and -independent manner. ESCC carcinogenesis involves inflammation in which the Stat3 signaling pathway is frequently activated. Given that Stat3 is a wellknown cancer therapeutic target, we investigated whether Stat3 is involved in metformin action in ESCC cells. Western blot analysis showed that expression of phosphorylated Stat3 and its target cyclin D1 were dramatically repressed by metformin treatment (Figure 5a). To further confirm the negative regulatory role of Stat3 signaling in autophagy in metformin-treated ESCC cells, we characterized the effects of metformin in cells in which Stat3 was silenced with siRNA (Supplementary Figure 2). In line with metformin treatment, knockdown of Stat3 not only induced apoptosis, as indicated by increased cleaved PARP (Figure 5b), but also resulted in autophagy, as evidenced by conversion of LC3, enhanced Beclin-1 expression and increased punctate GFP-LC-3 fluorescence intensity (Figures $5 b$ and $c$ ). Moreover, silencing of Stat3 resulted in enhanced metformin-induced decreases in cell viability, as assessed by MTT assay, compared with control (Supplementary Figure 3). Thus, these data suggest that Stat3 inactivation is involved in metformin-mediated inhibition of growth, and induced autophagy and apoptosis.

Next, we determined the potential mechanism by which Stat3 inactivation by metformin triggers apoptosis and autophagy in metformin-treated cells. It is noteworthy that $\mathrm{Bcl}-2$, a direct downstream target of Stat3, was repressed by 

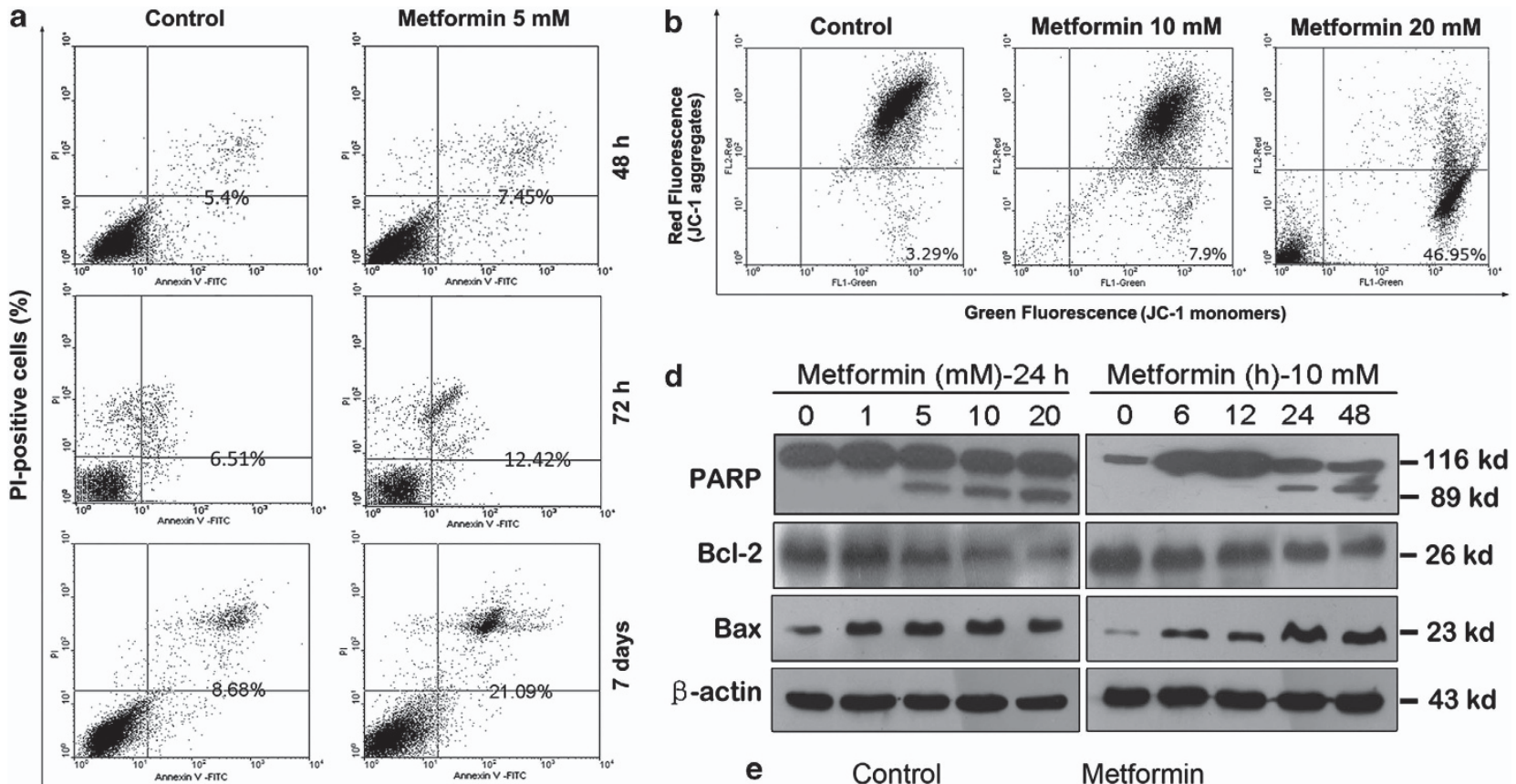

Green Fluorescence (JC-1 monomers)

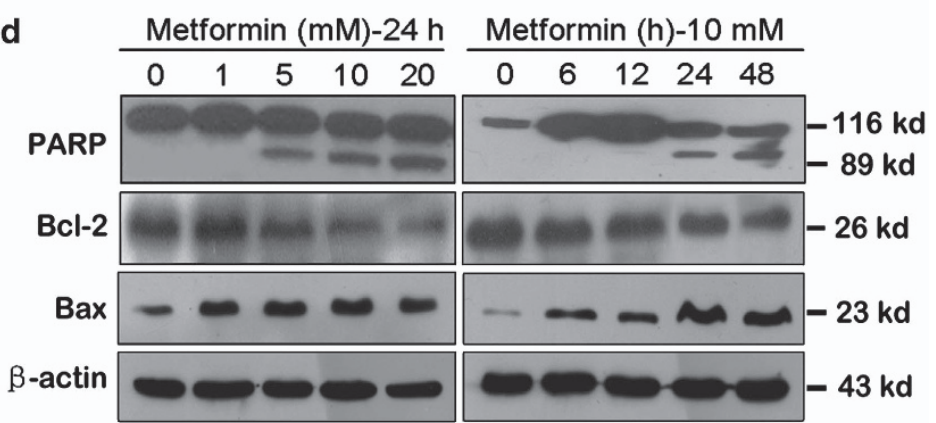

Annexin V-FITC-positive cells (\%)
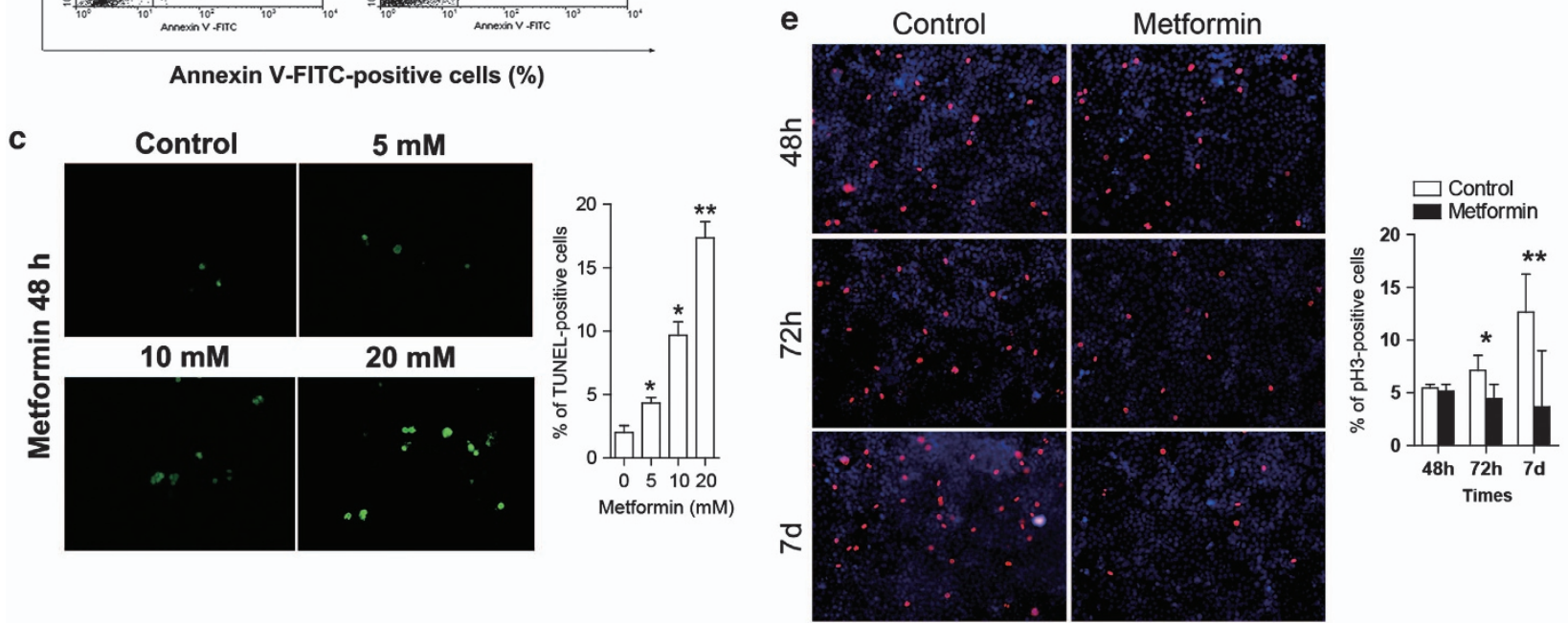

Figure 2 Metformin induces apoptotic cell death in ESCC cells. (a) Cells were stained with annexin-V-FITC $(20 \mu \mathrm{g} / \mathrm{ml})$ and PI (20 mg/l) following treatment with or without metformin for 48 and $72 \mathrm{~h}$ and for 7 days. Apoptosis was analyzed using flow cytometry. (b) Cells were stained with JC-1 (10 $\mu \mathrm{g} / \mathrm{ml})$ following treatment with or without metformin at the indicated concentrations for $48 \mathrm{~h}$. Mitochondrial membrane potential (MMP) was analyzed using flow cytometry. (c) TUNEL staining of EC109 cells after $48 \mathrm{~h}$ of metformin treatment at the indicated concentrations. Positive cells were labeled with TUNEL (green) (magnification, $\times 400$ ). Quantification of the number of TUNEL-positive cells in the control and metformin-treated cells (graph on the right), plotted as a percentage TUNEL-positive cells. ${ }^{*} P<0.05$ and ${ }^{* *} P<0.01$ represent significant differences compared with the control. The $P$-values were calculated using a two-sided Student's $t$-test. (d) Effects of metformin on apoptosis-related proteins assayed by western blot at different treatment concentrations (left) and different times (right). $\beta$-Actin was probed as the loading control. (e) Cell proliferation assessed by immunohistochemistry with antiphospho-Histone $\mathrm{H} 3$ (pH3) antibody in ESCC cells after treatment with metformin for 48 and $72 \mathrm{~h}$ and for 7 days. pH3 were red and nuclei were stained with DAPI (blue). Per field, 100 cells were counted, and 10 fields were counted per sample (magnification, $\times 200$ ). Graphs represent the percentage of pH3-positive cells. ${ }^{* \star} P<0.01$ and ${ }^{* * *} P<0.001$ compared with the control. $P$-values were calculated using a two-sided Student's $t$-test. Representative data from one of three independent experiments are shown in panels from (a) through (d)

metformin treatment (Figure 2d), whereas Bcl-2 was further downregulated by metformin in Stat3-silenced cells (Figure $5 \mathrm{~b}$ ). Bcl-2 is known to regulate both apoptosis and autophagy. ${ }^{21,28-30}$ Ectopic expression of Bcl-2 in EC109 cells, by transfection with a Bcl-2 expression plasmid, inhibited the upregulation of Bax and cleaved PARP expression by metformin (Figure $5 \mathrm{~d}$ ), indicating that $\mathrm{Bcl}-2$ overexpression can block metformin-induced apoptosis. Similarly, Bcl-2 overexpression also attenuated metformin-triggered autophagy, as evidenced by diminished LC3 conversion, and lack of
Beclin-1 and Atg5 upregulation (Figure 5d). These data indicate that metformin induces apoptosis and autophagy at least partially via inactivation of Stat3 and repression of Bcl-2.

Furthermore, to evaluate the clinical impact of Stat $3 / \mathrm{Bcl}-2$ signaling in ESCC, we characterized p-Stat3 and Bcl-2 expression in resected specimens from patients with ESCC. Immunohistochemistry $(\mathrm{IHC})$ showed that both $\mathrm{p}$-Stat3 and $\mathrm{BCl}-2$ were overexpressed in ESCC samples, compared with nontumor tissues, and both proteins positive correlated with each other (Figure 5e, $r=0.695$ and $P<0.001$ ). 
a

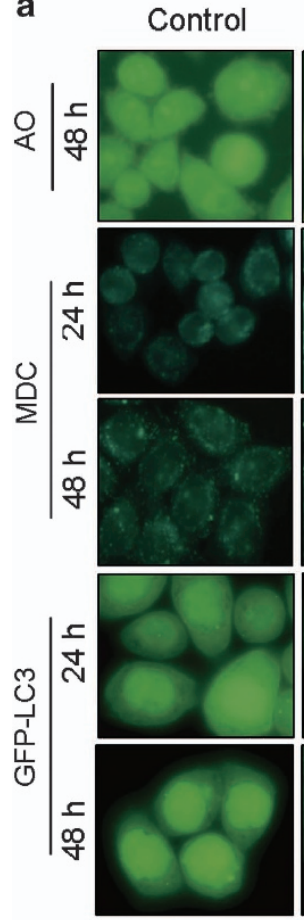

$10 \mathrm{mM}$
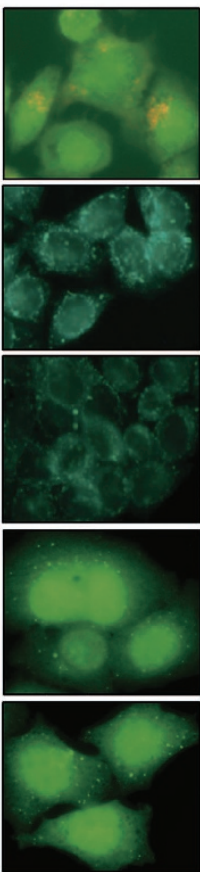

$20 \mathrm{mM}$
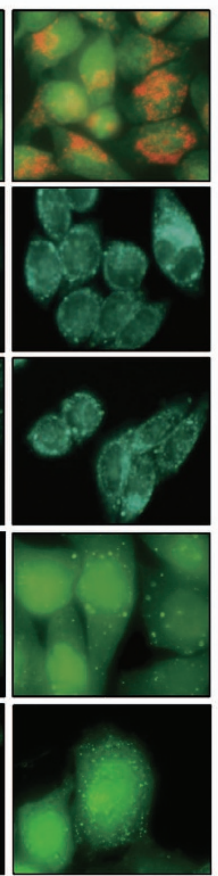

b

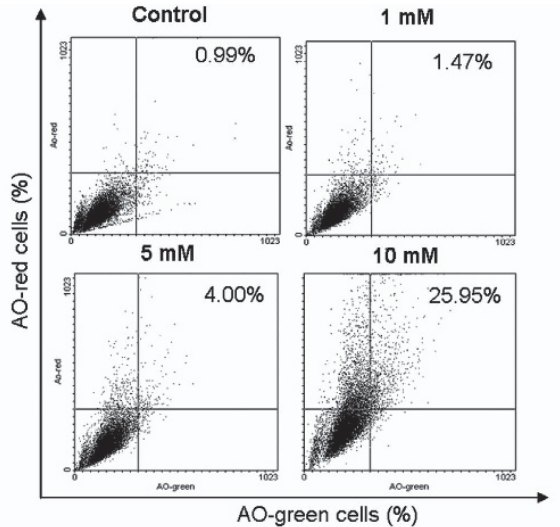

C

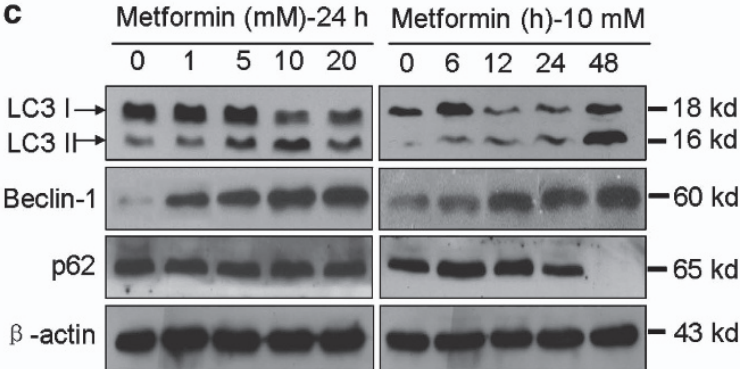

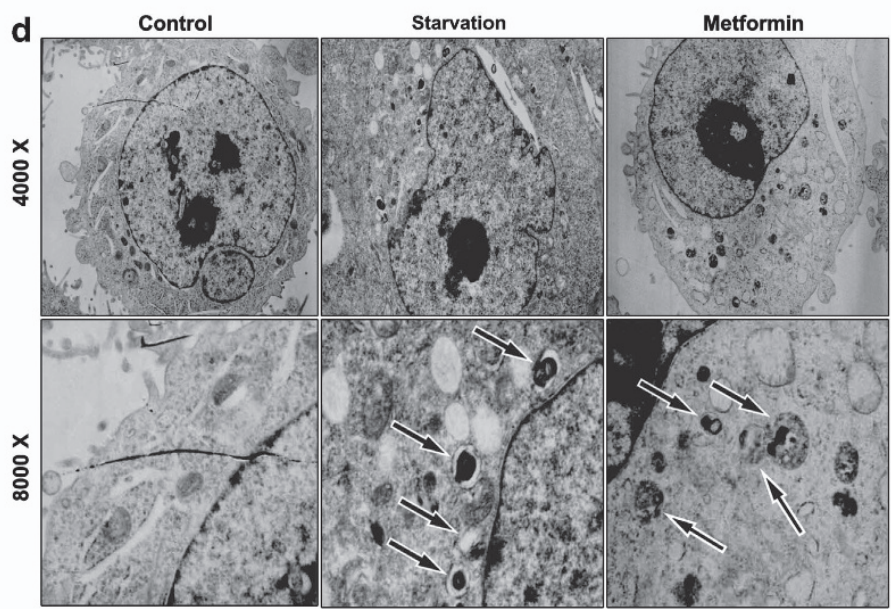

Figure 3 Metformin triggers ESCC cell autophagy. (a) Representative images of AO (upper) and MDC (middle) staining of ESCC cells following treatment with metformin at the indicated concentration for the indicated time. For AO staining (upper), red color intensity shows acidic vesicular organelles, representing autophagolysosomes. For MDC staining (middle), punctate fluorescence in the cytoplasm indicates formation of autophagic vacuoles. In the bottom panel, EC109 cells transfected with GFP-LC3 plasmid were treated with metformin for 24 and $48 \mathrm{~h}$. Control cells showed a diffuse expression pattern of GFP-LC3, whereas metformin-treated cells displayed GFP-LC3-Il a punctate pattern, indicating formation of autophagosomes. Magnification: $\times 400$ for AO and MDC stainings, and $\times 1000$ for GFP-LC3. (b) EC109 cells were treated with the indicated concentration of metformin for $48 \mathrm{~h}$, and then cells were stained with AO. Cell autophagy was analyzed by quantification of acidic vesicular organelles (AVOs) with $\mathrm{AO}$ using flow cytometry. (c) Effects of metformin on autophagy-related proteins assayed by western blot at different metformin concentrations (left) and different times (right). $\beta$-Actin was probed as the loading control. (d) Transmission electron microscopy (TEM) images showing autophagic vacuoles (arrows) observed in metformin-treated EC109 cells for $48 \mathrm{~h}$ (right). Starved cells were used as a positive control for autophagy (middle). No or few autophagic vacuoles are observed in control cells (left panel). Representative data from one of three independent experiments are shown in $(\mathbf{a}-\mathbf{c})$

AMP-activated protein kinase (AMPK) is a sensor of cellular and systemic energy homeostasis. ${ }^{31}$ Metformin activates AMPK to phosphorylate mammalian target of rapamycin (mTOR). ${ }^{31,32}$ However, metformin can exert its effects through both AMPKdependent and -independent mechanisms in different cellular settings. ${ }^{20}$ We therefore determined whether AMPK participates in the cytotoxic effects of metformin treatment. Metformin indeed increased p-AMPK, but decreased p-mTOR (Figure 5f). AMPK knockdown partially inhibited metformin-mediated autophagy, as evidenced by decreased conversion of LC3-I to LC3-II (Figure 5f). Meanwhile, inactivation of Stat3 by metformin was only marginally blocked by AMPK knockdown (Figure 5f). These data suggest a limited involvement of AMPK in metforminmediated Stat3 inactivation and autophagy. Together, metformin induces apoptosis and autophagy in ESCC largely via crosstalk between Stat3 and Bcl-2. 

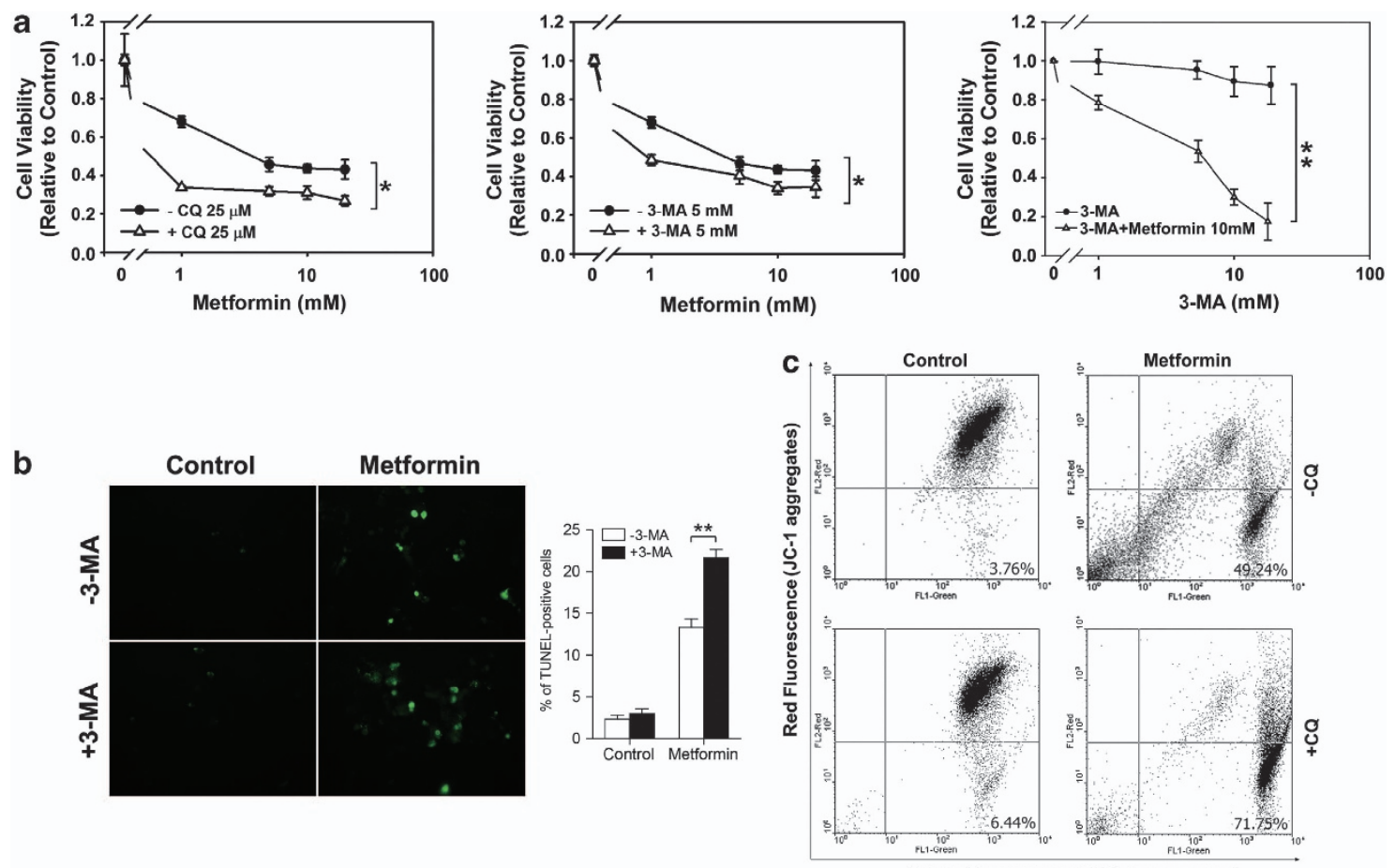

Green Fluorescence (JC-1 monomers)
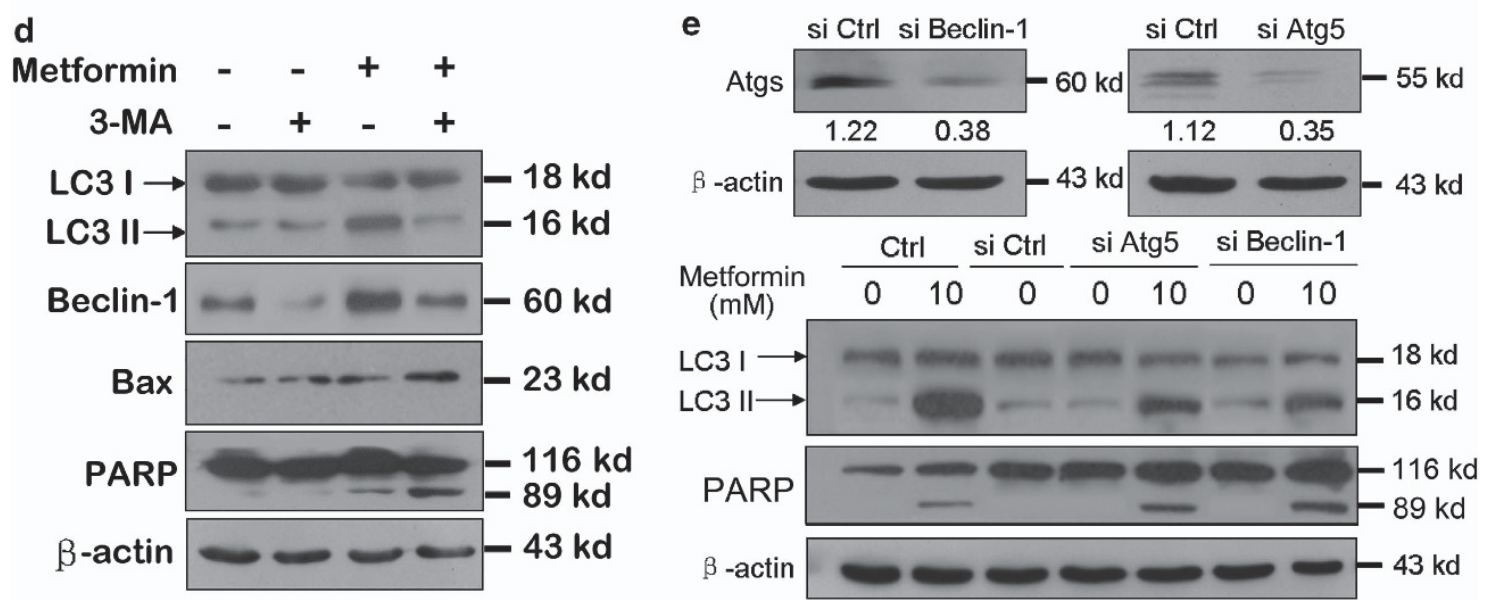

Figure 4 Inhibition of autophagy sensitizes cells to metformin-induced apoptotic cell death. (a) Inhibition of autophagy with 3-MA or CQ decreases viable metformintreated cells. Cell viability was measured by MTT assay after cells were incubated with the indicated concentration of metformin with or without 3-MA ( $\pm 3-\mathrm{MA}, 5 \mathrm{mM} ; \pm \mathrm{CQ}$, $25 \mu \mathrm{M}$ ) for $48 \mathrm{~h}$ (left and middle). Cell viability was measured by MTT assay after cells were incubated with the indicated concentration of 3-MA with or without metformin ( \pm metformin, $10 \mathrm{mM}$ ) for $48 \mathrm{~h}$ (right). Data were expressed as the means \pm SD from three separate experiments as the percentage of viable cells in the control group. ${ }^{*} P<0.05$ and ${ }^{* *} P<0.01$ by Student's $t$-test. (b) Representative images of TUNEL staining of EC109 cells after treatment with the indicated concentration of metformin with or without 3-MA ( $\pm 3-\mathrm{MA}$ ) for $48 \mathrm{~h}$ (magnification, $\times 400$ ). Quantification of the number of TUNEL-positive cells (graph on the right), plotted as a percentage TUNEL-positive cells. ${ }^{* \star} P<0.01$ by Student's $t$-test. (c) Cells were stained with $\mathrm{JC}-1(10 \mu \mathrm{g} / \mathrm{ml})$ following metformin treatment with or without metformin $\mathrm{CQ}( \pm \mathrm{CQ})$ for $48 \mathrm{~h}$. Mitochondrial membrane potential (MMP) was analyzed using flow cytometry. (d) Apoptosis- and autophagy-related proteins were examined by western blot following metformin treatment with or without 3-MA ( $\pm 3-\mathrm{MA}$ ) for $48 \mathrm{~h}$. (e) Effects of genetic inhibition of autophagy by knockdown of Beclin-1 or Atg5 on metformin-mediated apoptosis. LC3 and cleaved PARP were examined by western blot. Representative data from one of three independent experiments are shown in (b-e). Error bars indicate \pm S.E. ${ }^{*} P<0.05$ and ${ }^{\star *} P<0.01$ by Student's $t$-test. $\beta$-Actin was the loading control in (d) and (e)

Metformin inhibited ESCC tumor growth in vivo. We evaluated the effects of metformin in vivo in a xenograft tumor model by subcutaneous inoculation of EC109 cells into nude mice. On day 7 following tumor cell injection, mice were given daily intraperitoneal injections (i.p.) of metformin $(250 \mathrm{mg} / \mathrm{kg}$ body weight) for 4 weeks. Throughout the course of treatment, metformin did not cause visible side effects or change in body weight of the mice (Supplementary Figure 4). Consistent with in vitro results that metformin treatment decreased the growth of cultured ESCC cells, metformin administration was very effective in inhibiting tumor growth in vivo throughout the course of treatment (Figure 6a), resulting in decreased tumor size and weight (Figure 6b). These data indicate that metformin reduces tumor volume and growth rate of ESCC cells in vivo. 
a

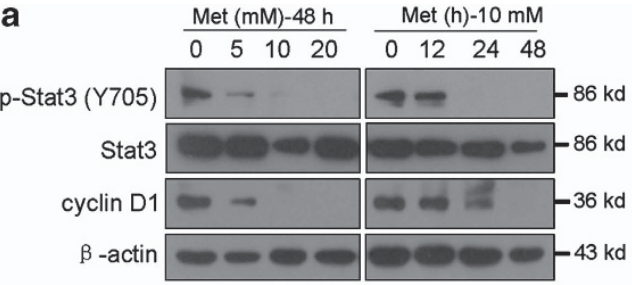

b

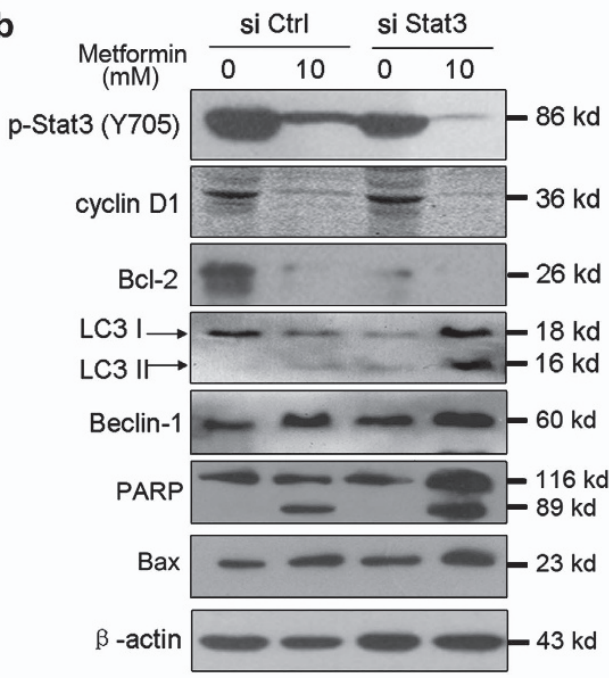

e

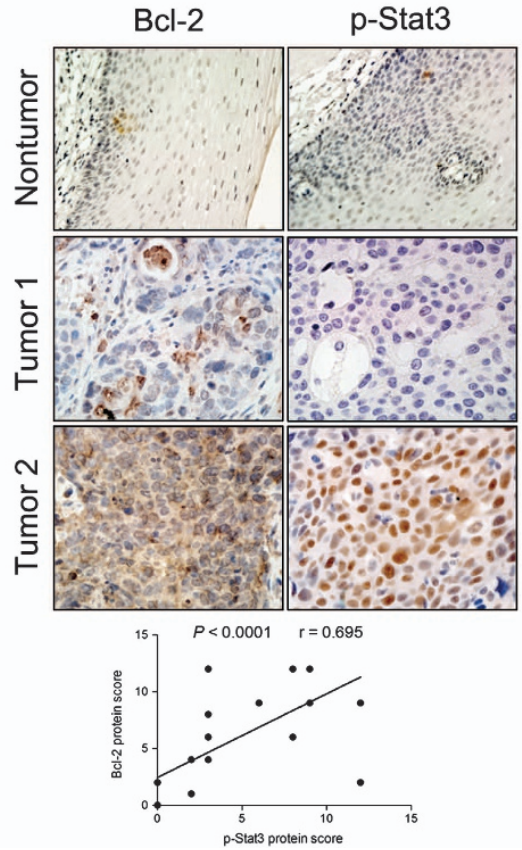

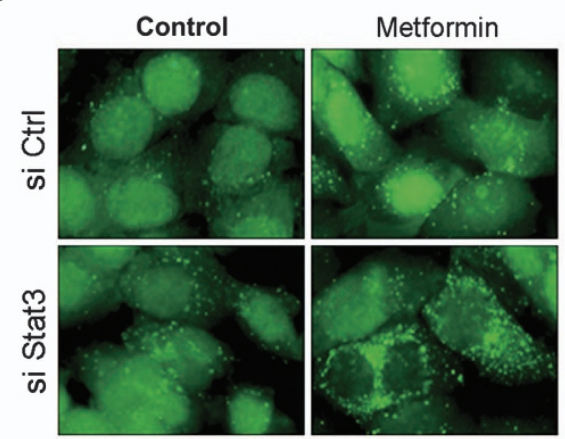

d
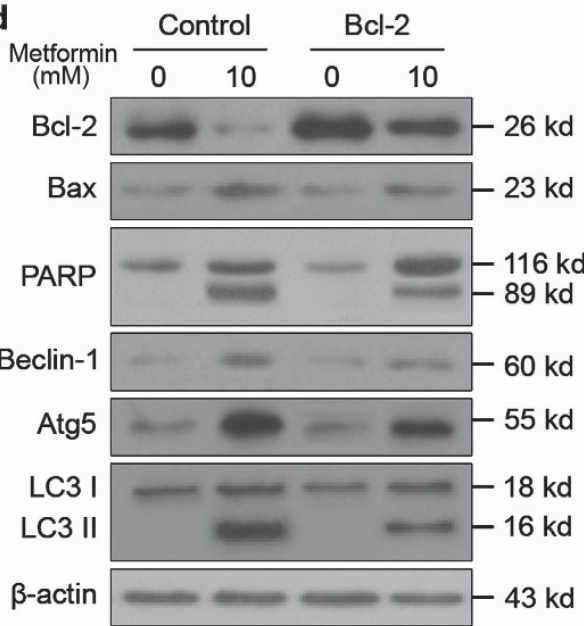

f

si Control si AMPK

Metformin

$\begin{array}{lllll}(\mathrm{mM}) & 0 & 10 & 0 & 10\end{array}$

p-AMPK $\longrightarrow-\infty-63 \mathrm{kd}$

AMPK $=-63 \mathrm{kd}$

LC3-I

LC3-II

p-mTOR

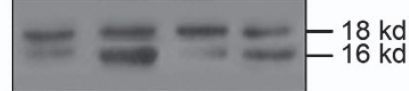

mTOR

p-Stat3

Stat3

$\beta$-actin

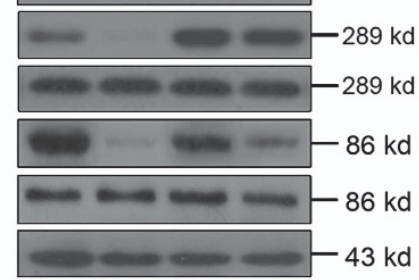

Figure 5 Inactivation of Stat3/Bcl-2 signaling contributes to metformin-induced effects. (a) Metformin treatment repressed expression of p-Stat3 and cyclin D1, as assayed by western blot. (b) Western blotting was used to assess p-Stat3, Stat3 targets (cyclin D1 and Bcl-2), apoptosis-related proteins, and autophagy-related proteins in Stat3 siRNA - or control siRNA-transfected EC109 cells treated with metformin. (c) Representative images ( $\times 1000)$ of GFP-LC3 expression patterns in Stat3 siRNA and control siRNA cells following metformin treatment. EC109 cells transfected with GFP-LC3 plasmid were used. (d) Ectopic expression of Bcl-2 attenuates metformin-mediated autophagy and apoptosis, as indicated by related protein expression assayed by western blot. (e) Representative images of IHC staining of $\mathrm{p}$-Stat3 and Bcl-2 in adjacent nontumor $(\times 200)$ and ESCC tumor tissues $(\times 400)$ from patients. The $p$-Stat3 protein level positively correlated with the Bcl-2 protein level in ESCC tumors from 36 patient samples (Pearson's correlation coefficient test, $r=0.695$ and $P<0.001$ ). (f) Effects of metformin treatment ( $10 \mathrm{mM}$ for $48 \mathrm{~h}$ ) on expression of AMPK/mTOR, $p$-Stat3 and LC3, as assayed by western blot, following transfection with AMPK siRNA or control siRNA. Representative data from one of three independent experiments are shown in (a, $\mathbf{b}, \mathbf{c}, \mathbf{d}$ and $\mathbf{f})$. $\beta$-Actin was applied as the loading control for $(\mathbf{a}, \mathbf{b}, \mathbf{d}$ and $\mathbf{f})$ 

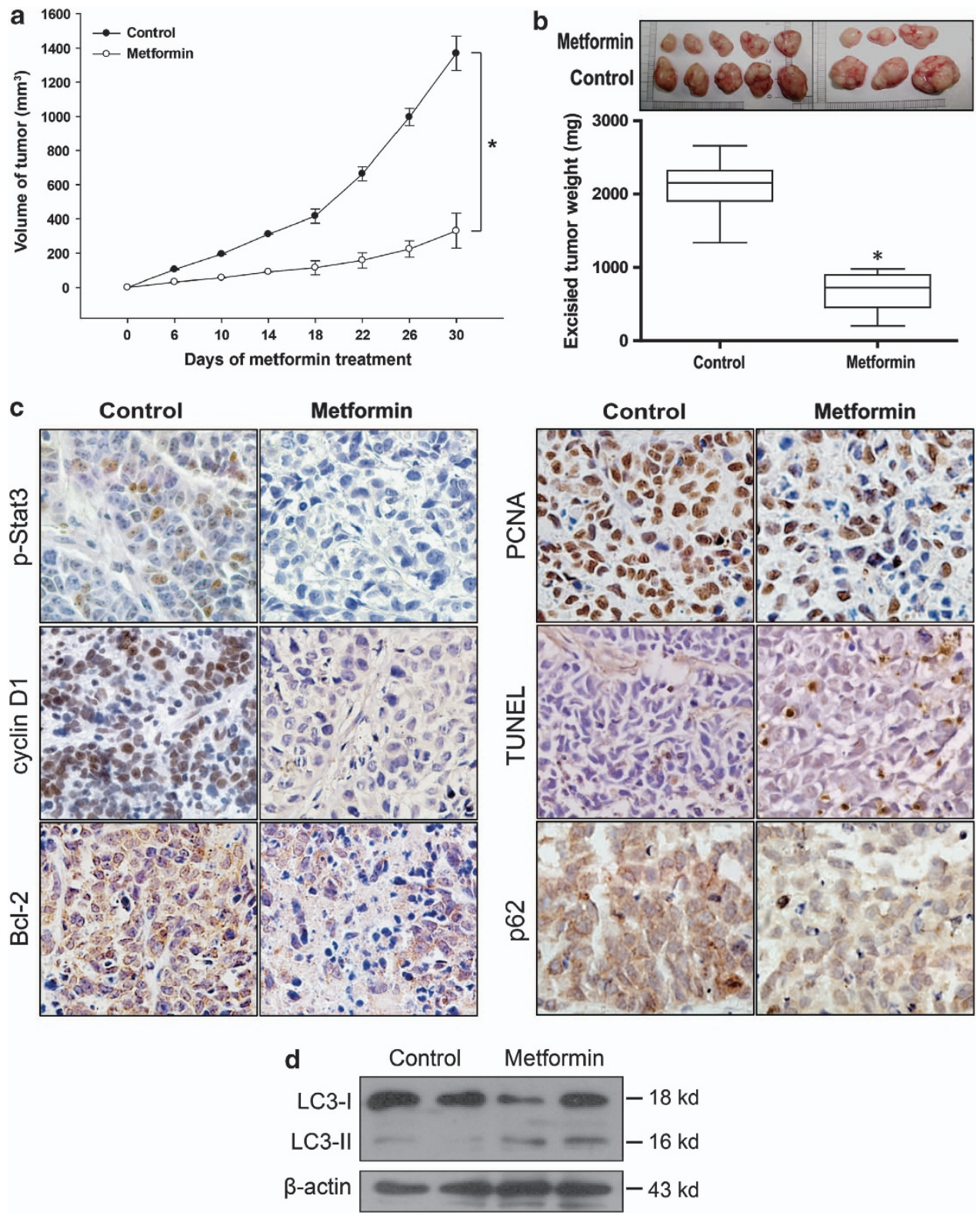

Figure 6 Metformin inhibits ESCC tumor growth in vivo. (a) Tumor growth curve of metformin-treated mice ( $250 \mathrm{mg} / \mathrm{kg}$ body weight) and control (vehicle treated) mice. Data represent the mean \pm S.D. of eight mice in each group. ${ }^{*} P<0.05$ by the Student's $t$-test. (b) Dissected tumor was photographed and weight was measured. ${ }^{*} P<0.05$ by Student's $t$-test. (c) Representative images $(\times 400)$ of IHC analysis of p-Stat3, cyclin D1, Bcl-2, PCNA, TUNEL and p62 in tumors. (d) Western blots of LC3 in tumor tissues. $\beta$-Actin was probed as the loading control

TUNEL analysis showed that metformin increased apoptosis (Figure 6c). Tumors exhibited decreased p62 IHC, whereas LC3 conversion was enhanced, as determined by western blot, indicating increased autophagy following metformin administration (Figures $6 \mathrm{c}$ and d). Furthermore, IHC using an antibody against PCNA, a marker of cell proliferation, showed that PCNA-positive cells were decreased in metformin-treated mice as compared with control mice (Figure 6c), demonstrating decreased cell proliferation induced by metformin treatment (Figure 6c).

We also investigated whether metformin affects Stat3/Bcl-2 signaling of human ESCC cells in vivo. IHC revealed decreased expression of $\mathrm{p}$-Stat3, and two Stat3 targets, cyclin D1 and $\mathrm{Bcl}-2$, in tumors from mice treated with metformin, as compared with control mice (Figure 6c), whereas total Stat3 levels remained unchanged (data not shown). These data indicate metformin treatment inactivates Stat3/Bcl-2 activity in vivo.

To confirm the cytoprotective action of metformin-mediated autophagy in vivo, mice were treated with both metformin and CQ. Co-treatment reduced tumor volume compared with metformin alone (Supplementary Figure 5). Consistent with our in vitro results, in vivo metformin administration inhibited ESCC cell growth through the integrative effects of apoptosis, autophagy and cell proliferation as a consequence of metformin-mediated inactivation of Stat3/Bcl-2 pathway. 


\section{Discussion}

This study describes the antineoplastic effects of metformin on ESCC cells in vitro and in vivo. We also propose a novel mechanism in which metformin inactivates Stat3 and subsequently represses $\mathrm{Bcl}-2$ to induce autophagy and apoptosis (Figure 7).

Although metformin has been studied in clinical trials for many types of cancers, its use in clinical trials for ESCC has not been considered. Recently, metformin was reported to increase response to chemoradiation in patients with esophageal adenocarcinoma, suggesting a therapeutic effect of metformin in patients with esophageal neoplasia. ${ }^{33}$ Although a previous report demonstrates that metformin inhibits proliferation of ESCC cells (KYSE30 and KYSE70) in vitro, ${ }^{34}$ our study corroborates and extends these studies to the roles of autophagy and apoptosis and to an in vivo ESCC model.

Both apoptosis and autophagy are crucial mechanisms regulating cell survival and homeostasis. ${ }^{17,19,35}$ Although modulation of autophagy by apoptotic signaling pathways has been studied extensively, it is also known that autophagy modulates apoptosis. ${ }^{5,17,19,35}$ Given that pharmacological or genetic inhibition of autophagy leads to increased metformininduced apoptosis, our present data argue that metforminmediated autophagy is a prosurvival mechanism rather than a cell death mechanism. This supports a prior study in which inhibition of autophagy sensitized apoptosis-resistant tumor cells to chemotherapy in chronic myeloid leukemia (CML) and lymphoma. ${ }^{31}$ In contrast, autophagy inhibition by silencing LC3 or Atg5 decreases apoptosis and inhibits the metformin inhibitory effects on melanoma cells. ${ }^{20}$ Although the circumstances under which autophagy functions as a primary cell death mechanism or survival remain to be defined, it is hypothesized that autophagy inhibition promotes apoptosis in cancer cells with intact apoptotic signaling pathways. ${ }^{36}$ However, the exact role and mechanism of metformininduced autophagy in different cancer types and different stages is worthy of further study.

The mechanisms of metformin inhibition are postulated to be associated with both indirect and direct effects of the drug. Indirect effects are linked to the ability of metformin to inhibit the transcription of key gluconeogenesis genes in the liver and stimulate glucose uptake in muscle, thus increasing insulin sensitivity and reducing blood glucose and lowering insulin levels. ${ }^{1,18}$ The direct effects of metformin are believed to be primarily mediated by activation of AMPK, leading to a reduction in mTOR signaling and protein synthesis in cancer cells. ${ }^{19,31,32}$ Despite these common mechanisms, cancer type-specific pathways may provide disease-specific targets and relevant biomarkers in clinical settings. In this case, ESCC is a malignancy associated with inflammation, in which Stat3 and its direct targets, such as Bcl-2 and cyclin D1, are frequently disregulated. ${ }^{11,14,37}$ Our in vitro and in vivo findings suggest that metformin exerts antineoplastic activities through downregulation of Stat3 signaling; that is, Stat3 modulates the autophagic process induced by metformin in addition to its participation in cell proliferation and apoptosis. The regulation by Stat3 of autophagy, defined in our study, is also supported by similar publications in triple-negative breast cancer, ${ }^{19}$ malignant glioma, ${ }^{38}$ Hela cells ${ }^{39}$ and cardiac disease. ${ }^{40}$

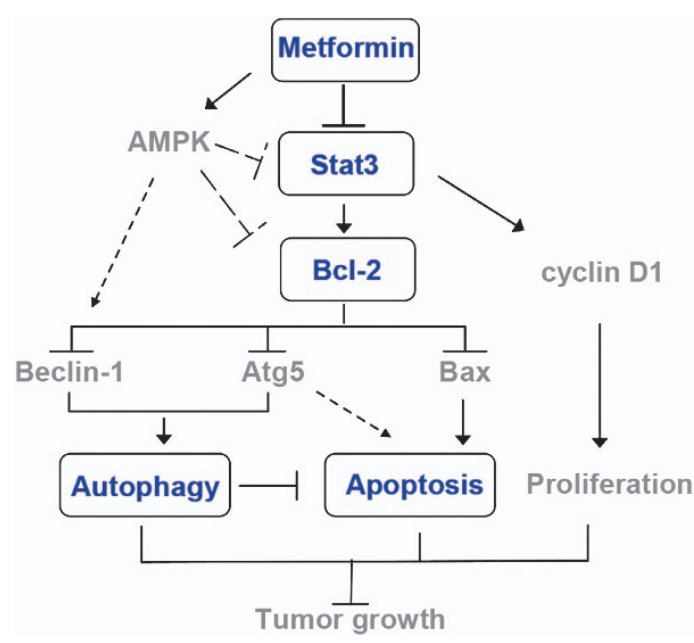

Figure 7 Proposed mechanisms responding to metformin-induced effects in ESCC

We further investigated the possible mechanism of how Stat3 triggers apoptosis and autophagy. In this study, Bcl-2, a pro-survival gene directly induced by Stat $3,{ }^{21,41}$ is repressed by metformin treatment. Furthermore, siRNA-mediated knockdown of Stat3 exacerbates metformin-mediated Bcl-2 repression. Prior reports have shown that inactivation of Stat3 correlates with altered $\mathrm{Bcl}-2 / \mathrm{Bax}$ expression and induction of apoptosis. ${ }^{41}$ Likewise, our findings indicate that Stat3 mediates apoptosis through regulation of Bcl-2 in ESCC cells following metformin treatment.

Few studies have directly defined the mechanism of how Stat3 triggers autophagy in cancer cells. Bcl-2 is also known to negatively regulate Beclin-1-dependent autophagy both in vitro and in vivo. ${ }^{21,28} \mathrm{An}$ increasing body of data support the hypothesis that $\mathrm{Bcl}-2$ prevents cells from undergoing autophagy by binding and inhibiting Beclin-1. ${ }^{21,28}$ Therefore, repression of $\mathrm{Bcl}-2$ may dissociate the $\mathrm{Bcl}-2 / \mathrm{Beclin}-1$ complex and thereby increase cellular autophagy. ${ }^{30}$ It is also evident that Bcl-2 siRNA induces expression of Beclin-1 and Atg5 autophagy-promoting proteins in breast cancer cells, ${ }^{28}$ suggesting that inhibition of $\mathrm{Bcl}-2$ induces autophagy through induction of Beclin-1 and Atg5. In our study, downregulation of Bcl-2 by metformin treatment/Stat3 inactivation leads to liberated or induced Beclin-1/Atg5, thereby triggering autophagy. This observation was further confirmed by showing that ectopic expression of $\mathrm{Bcl}-2$ in ESCC cells attenuated metformin-induced autophagy. We further describe that Bcl2 overexpression positively correlates with p-Stat3 in ESCC samples of patients (Figure $5 \mathrm{e}$ ). In addition, we also show that metformin treatment of mice bearing ESCC tumors resulted in decreased levels of $\mathrm{Bcl}-2$ along with decreased $\mathrm{p}$-Stat3, and inhibited tumor growth. Given that knockdown $\mathrm{Bcl}-2$ has been proposed as a therapeutic strategy alone or with chemotherapy in cancers that overexpress Bcl-2, ${ }^{28}$ Stat3-Bcl-2 proto-oncogene pathway may provide promising opportunities as therapeutic targets for ESCC.

AMPK is an important sensor of energy balance. Metformin treatment activates AMPK both in vivo and in vitro. Metforminmediated AMPK activation was also observed in our study. 
However, AMPK activation does not play a prominent role in metformin-mediated Stat3 inactivation, as metformin robustly inhibits the Stat3 pathway in AMPK-silenced cells, although we cannot exclude direct regulation of cell survival and cell death by AMPK (to Beclin for example) or through other pathways that may also contribute to cell growth and survival (Figure 7).

Collectively, metformin exerts antineoplastic effects on ESCC cells in vitro and in vivo. These beneficial effects can be explained by metformin-mediated Stat3 inactivation and $\mathrm{Bcl}-2$ repression with downstream induction of apoptosis and autophagy. In a translational setting, our work motivates future clinical trials to investigate whether inhibition of autophagy or blockage of Stat3/Bcl-2 function may enhance the antineoplastic effects of metformin in human ESCC.

\section{Materials and Methods \\ Reagents and antibodies. Metformin (D150959), CQ (C6628), MDC (30432), 3-MA (M9281), 4',6-diamidino-2- phenylindole (DAPI; D9542), antibodies against anti- $\beta$ and LC3, anti-rabbit secondary antibody, anti-mouse secondary antibody and Cy3-labeled rabbit secondary antibody were purchased from Sigma (St. Louis, MO, USA). Antibodies against p-Stat3 (Y705), Stat3, cyclin D1, LC3, PCNA and PARP were purchased from Cell Signaling (Beverly, MA, USA); antibodies against p-AMPK, p-mTOR, Beclin-1, Bcl-2, p62 and Bax were purchased from Santa Cruz (Santa Cruz, CA, USA). Anti-phospho-Histone H3 (Ser10) antibody was purchased from Upstate (Millipore, Billerica, MA, USA). Dulbecco's modified Eagle's medium (DMEM) and fetal bovine serum were obtained from Gibco (Life Technologies Gibco/BRL, New York, NY, USA). Crystal violet staining and JC-1 were obtained from Beyotime Institute of Biotechnology (Shanghai, China). ApopTag plus peroxidase in situ apoptosis detection kit was obtained from Millipore. AO was obtained from Poly-Sciences (Warrington, PA, USA). Stat3, AMPK, Beclin-1 and Atg5 siRNAs were purchased from Shanghai GenePharma (Shanghai, China). Lipofectamine 2000 was bought from Invitrogen} (Carlsbad, CA, USA).

Human tissue specimen. In this study, the paraffin-embedded pathological specimens from 36 patients with ESCC and adjacent nontumor tissues were obtained from Cancer Hospital of Shantou University Medical College in the Chaoshan littoral, which is located in Southern China and is recognized as one of the high-incidence regions with esophageal cancer, between March and September 2009. This study was approved by the Institutional Review Board and the Ethics Committee of Cancer Hospital of Shantou University Medical College (IRB serial number: \#04-070).

Cell culture and cell viability assay and colony formation assay. EC109 and EC9706 cells were obtained from the tumor cell bank of the Chinese Academy of Medical Science ${ }^{5,8}$ and were cultured in DMEM medium supplemented with $10 \% \mathrm{FBS}, 100 \mathrm{unit} / \mathrm{ml}$ penicillin and $100 \mathrm{unit} / \mathrm{ml}$ streptomycin at $37^{\circ} \mathrm{C}$ in a humidified atmosphere with $5 \% \mathrm{CO}_{2}$. Immortalized NE3 cells were cultured in Defined Keratinocyte-SFM (DK-SFM)/Epilife mixed medium (Life Technologies Gibco/BRL).,42 All experiments were performed during the exponential phase of cell growth.

Cell viability was assessed by MTT assay as described previously. ${ }^{8}$ Cells were plated in 96-well plates at a density of 5000 cells in $100 \mu$ l medium per well 1 day before the experiment. The cells were treated with metformin with indicated condition including co-treatment with 3-MA or $\mathrm{CQ}$, gene knockdown and gene overexpression. The cell viability was examined by MTT assay.

For colony formation assay, following treatment, adherent cells were trypsinized and 1000 viable cells were subcultured in six-well plates (in triplicate). Cells were allowed to adhere and colonize for 14 days. To visualize colonies, media were removed and cells were fixed in $96 \%$ ethanol for $10 \mathrm{~min}$ and stained with crystal violet staining solution.

Western blot analysis. Whole-cell lysates and tissue lysates were prepared from treated cells and xenograft tumor for immunoblotting analysis as described previously. ${ }^{43}$ Protein extraction and western blot analysis were performed as previously. ${ }^{44}$ Immunoblotting was performed on the nitrocellulose membranes (Millipore) using enhanced chemiluminescence (ECL kit, Pierce, Thermo Fisher Scientific, Waltham, MA, USA). The blots were visualized by autoradiography for cyclin D1 (Figure $5 \mathrm{~b}$ ). For all other proteins, membranes were probed with anti-rabbit secondary antibodies conjugated to IRdye 800 (Rockland Immunochemicals, Gilbertsville, PA, USA) and blotted proteins were detected and quantified using the Odyssey infrared imaging system (Li-Cor Biosciences, Lincoln, NE, USA).

GFP-LC3 analysis. EC109 cells stably expressing GFP-LC3 were obtained by transfecting the cells with pEGFP-LC3 plasmid and selected with G418. Transfection using Lipofectamine 2000 Reagent (Invitrogen) was carried out according to the manufacturer's instructions. After transfection $(5 \mathrm{~h})$, cells were washed twice with phosphate-buffered saline, and fresh DMEM was added for further incubation. The formation of GFP-LC3 punctate structures was examined as previously described. ${ }^{31}$ The images were taken using the fluorescence microscope (Olympus IX70, Olympus, Tokyo, Japan).

Immunohistochemistry, immunofluorescence and TUNEL assay. IHC staining was performed as previously described. ${ }^{44}$ Briefly, $4 \mu \mathrm{m}$ sections were cut from specimens that had been fixed in $10 \%$ buffered formalin and embedded in paraffin. After undergoing deparaffinization and rehydration, endogenous peroxidase blocking and antigen retrieval, specimens were incubated overnight at $4^{\circ} \mathrm{C}$ with anti-p-Stat3 polyclonal antibody $(1: 200)$, anti-Stat3 polyclonal antibody (1:200), anti-cyclin D1 polyclonal antibody, anti-Bcl-2 polyclonal antibody (1:100), anti-p62 polyclonal antibody $(1: 200)$ or anti-PCNA polyclonal antibody $(1: 200)$. Staining was visualized using an EnVision antibody complex method; an EnVision kit (ZSGB-BIO, Beijing, China) was employed and $3,3^{\prime}$-diaminobenzidine was used as the chromogen. Nuclei were counterstained with hematoxylin. Positive controls were sections from a breast cancer. Sections immunostained with rabbit or mouse $\lg G$ as the primary antibody were used as negative controls. Immunostaining results were graded semiquantitatively by counting the percentage of positive cells and intensity. The specimens were evaluated by two independent observers who were unaware of the clinical information.

For immunofluorescence assay of $\mathrm{pH} 3$, fixed cells were permeabilized with $0.1 \%$ Triton X-100 at room temperature for 15 min and incubated with anti-pH3 antibody overnight at $4^{\circ} \mathrm{C}$. Cells were washed 3 times with PBST (phosphate-buffered saline with Tween-20) and then incubated for $1 \mathrm{~h}$ with Cy3-conjugated goat anti-rabbit IgG at room temperature. For identification of cells with nuclear changes typical of apoptosis, cells were stained with $0.1 \mu \mathrm{g} / \mathrm{ml}$ DAPI for $5 \mathrm{~min}$. Samples were examined under a fluorescence microscope (Olympus IX70).

TUNEL assay was performed on cells and tumor tissues from mice. Apoptotic cells were detected using ApopTag plus peroxidase in situ apoptosis detection kit according to the manufacturer's instructions. ${ }^{45}$ Stained sections were visualized under fluorescence microscope.

Analysis of apoptosis by Annexin V-FITC and by JC-1. Apoptosis was quantified with an Annexin V-FITC apoptosis detection kit (Beyotime Institute of Biotechnology) as described by the manufacturer's instructions. After exposure to drugs for $48 \mathrm{~h}$, the cells were collected and washed with PBS, gently resuspended in Annexin V binding buffer and incubated with Annexin V-FITC/PI. Flow cytometry was performed using Cellquest software (BD Biosciences, San Jose, CA, USA).

Mitochondrial membrane potential $(\Delta \Psi \mathrm{m})$ changes were investigated using JC-1, a sensitive fluorescent probe for $\Delta \Psi \mathrm{m} .{ }^{5}$ Cells were stained with $5 \mu \mathrm{M} \mathrm{JC}-1$ for $30 \mathrm{~min}$ at $37^{\circ} \mathrm{C}$. After rinsing with ice-cold PBS, cells were resuspended in PBS and immediately assessed by flow cytometry for red and green fluorescence. A $488 \mathrm{~nm}$ filter was used for the excitation of JC-1. Emission filters of 535 and $595 \mathrm{~nm}$ were used to quantify the population of mitochondria with green (JC-1 monomers) and red (JC-1 aggregates) fluorescence. Frequency plots were prepared for FL1 and FL2 (red) to determine the percentage of the mitochondria that stained green (low membrane potential) versus red (normal membrane potential).

Autophagy analysis by immunofluorescence and flow cytometry. Assessment of autophagy was accomplished by detecting and quantifying AVOs using immunofluorescence (AO staining and MDC staining) and flow cytometry.

Cell staining with $\mathrm{AO}$ was performed as previously. ${ }^{5,46}$ Treated cells were stained with $\mathrm{AO}$ at a final concentration of $1 \mathrm{mg} / \mathrm{ml}$ for $17 \mathrm{~min}$. Photographs were obtained with a fluorescence microscope (Olympus IX70). AO-stained cells were quantified using flow cytometry. 
For MDC staining, ${ }^{5,46}$ autophagic vacuoles of treated cells were stained with MDC by incubating cells with $0.05 \mathrm{mM} \mathrm{MDC}$ at $37^{\circ} \mathrm{C}$ for $30 \mathrm{~min}$. After incubation, cells were washed and immediately analyzed with a fluorescence microscope (Olympus IX70).

Transmission electron microscopy. TEM assay was performed as described previously. ${ }^{5}$ EC109 cells were harvested by trypsinization and fixed with ice-cold glutaraldehyde. The cells were then fixed in $\mathrm{OsO}_{4}$ and embedded in Epon-812. Fixed cells were sliced into $1 \mu \mathrm{m}$ sections and stained with uranium. Representative areas were chosen for ultra-thin sections to view with a JEM-1400 electron microscope (JEOL Ltd., Tokyo, Japan).

Gene knockdown using siRNA. The siRNAs to Atg5, Beclin-1, Stat3, AMPK or control siRNA were all purchased from Shanghai GenePharma. Cells were transfected with siRNA using Lipofectamine 2000 (Invitrogen) according to the manufacturer's instructions. Cells were incubated for $48 \mathrm{~h}$ before further treatment.

EC109 with ectopic expression of Bcl-2. A plasmid harboring Bcl2 cDNA or control was introduced into EC109 cells, using Lipofectamine 2000 Reagent (Invitrogen) according to the manufacturer's protocol. After 24-h transfection, the medium was replaced and cells with transient expression of Bcl-2 were treated with $10 \mathrm{mM}$ metformin for indicated periods of time. Cell lysates were collected and subjected to western blot analysis.

Animal studies. All animal experiments were done according to an Institutional Animal Care and Use Committee-approved protocol. Institutional guidelines for the proper and humane use of animals in research were followed. Female nude mice (nu/nu, 5-6 weeks old) were purchased from Beijing Vital River Laboratory Animal, Inc. (Beijing, China) and maintained under specific pathogenfree conditions in this study. Before injecting into animals, EC109 cells were washed twice and counted, and $1.5 \times 10^{6}$ cells suspended in $100 \mu \mathrm{l}$ of PBS were inoculated subcutaneously in the flanks. Treatment with metformin was started 7 days after inoculation of the cells. The experimental group $(n=8)$ was treated daily with i.p. injections of metformin $(250 \mathrm{mg} / \mathrm{kg}$ body weight) for the next 4 weeks until the mice were killed, whereas the control group $(n=8)$ received equal volume of vehicle only. For $C Q$ and metformin co-treatment experiment, the experimental group $(n=5)$ was treated daily with i.p. injections of metformin (250 mg/kg body weight) and CQ (250 mg/kg body weight) for the next 4 weeks until the mice were killed, whereas the control group $(n=5)$ received equal volume of metformin only. Throughout the course of treatment, the mice were monitored daily for any discomfort and weighed every third day to check for physical condition. The length and width of the tumors were measured using calipers twice per week for 4 weeks and tumor volume was calculated using the formula: length $\times(\text { width })^{2} \times 0.52$, as described previously. ${ }^{45}$

Statistical analysis. All statistical analyses were performed using the SPSS 17.0 statistical software package (SPSS Inc., Chicago, IL, USA). Comparisons between two groups were performed using Student's $t$-test. Bivariate correlations between study variables were calculated by Pearson's correlation coefficients. Data represent the means \pm S.D. The $P$-value of $<0.05$ was considered statistically significant.

\section{Conflict of Interest}

The authors declare no conflict of interest.

Acknowledgements. This work was supported by National Natural Science Foundation of China (81071736 and 30973508) and the Research Fund for the Doctoral Program of Higher Education of China (RFDP, 20104402110005). The immortalized NE3 cells was a kind gift from Dr. SW Tsao at Hong Kong University. We thank Dr. Xiao-kun Zhang (Burnham Institute, USA) and Dr. Jinzhang Zeng (Xiamen University, China) for kindly providing plasmids (pEGFPLC3 and Bcl-2). We thank Lin Zheng, Jinfeng Gan and Hongcai Chen for their technical assistance. We thank Dr. Stanley Lin for proofreading.

1. Pollak MN. Investigating metformin for cancer prevention and treatment: the end of the beginning. Cancer Discov 2012; 2: 778-790.
2. Taubes G. Cancer research. Cancer prevention with a diabetes pill? Science 2012; 335: 29

3. Goodwin PJ, Stambolic V, Lemieux J, Chen BE, Parulekar WR, Gelmon KA et al. Evaluation of metformin in early breast cancer: a modification of the traditional paradigm for clinical testing of anti-cancer agents. Breast Cancer Res Treat 2011; 126: 215-220.

4. Hadad S, Iwamoto T, Jordan L, Purdie C, Bray S, Baker L et al. Evidence for biological effects of metformin in operable breast cancer: a pre-operative, window-of-opportunity, randomized trial. Breast Cancer Res Treat 2011; 128: 783-794.

5. Tang Q, Li G, Wei X, Zhang J, Chiu J-F, Hasenmayer D et al. Resveratrol-induced apoptosis is enhanced by inhibition of autophagy in esophageal squamous cell carcinoma. Cancer Lett 2013; 336: 325-337.

6. Kamangar F, Dores GM, Anderson WF. Patterns of cancer incidence, mortality, and prevalence across five continents: defining priorities to reduce cancer disparities in different geographic regions of the world. J Clin Oncol 2006; 24: 2137-2150.

7. Zhang H, Lin W, Kannan K, Luo L, Li J, Chao P-W et al. Aberrant chimeric RNA GOLM1MAK10 encoding a secreted fusion protein as a molecular signature for human esophageal squamous cell carcinoma. Oncotarget 2013; 4: 2135-2143.

8. You YJ, Chen YP, Zheng XX, Meltzer SJ, Zhang H. Aberrant methylation of the PTPRO gene in peripheral blood as a potential biomarker in esophageal squamous cell carcinoma patients. Cancer Lett 2012; 315: 138-144.

9. Taccioli C, Chen H, Jiang Y, Liu XP, Huang K, Smalley KJ et al. Dietary zinc deficiency fuels esophageal cancer development by inducing a distinct inflammatory signature. Oncogene 2012; 31: 4550-4558.

10. Zhao YQ, Schetter AJ, Yang GB, Nguyen G, Mathe EA, Li $P$ et al. microRNA and inflammatory gene expression as prognostic marker for overall survival in esophageal squamous cell carcinoma. Int J Cancer 2013; 132: 2901-2909.

11. Timme S, Inde S, Fichter CD, Waehle V, Bogatyreva L, Atanasov $\mathrm{K}$ et al. STAT3 expression, activity and functional consequences of STAT3 inhibition in esophageal squamous cell carcinomas and Barrett's adenocarcinomas. Oncogene 2013; e-pub ahead of print 5 August 2013; doi:10.1038/onc.2013.298.

12. Yu H, Pardoll D, Jove R. STATs in cancer inflammation and immunity: a leading role for STAT3. Nat Rev Cancer 2009; 9: 798-809.

13. Yu H, Jove R. The STATs of cancer-new molecular targets come of age. Nat Rev Cancer 2004; 4: 97-105

14. Chen $\mathrm{X}$, Ying $\mathrm{Z}$, Lin $\mathrm{X}$, Lin $\mathrm{H}, \mathrm{Wu} \mathrm{J}$, Li $\mathrm{M}$ et al. Acylglycerol kinase augments JAK2/STAT3 signaling in esophageal squamous cells. J Clin Invest 2013; 123: 2576-2589.

15. Catlett-Falcone R, Landowski TH, Oshiro MM, Turkson J, Levitzki A, Savino R et al. Constitutive activation of Stat3 signaling confers resistance to apoptosis in human U266 myeloma cells. Immunity 1999; 10: 105-115.

16. Fukada T, Hibi M, Yamanaka Y, Takahashi-Tezuka M, Fujitani Y, Yamaguchi T et al. Two signals are necessary for cell proliferation induced by a cytokine receptor gp130: involvement of STAT3 in anti-apoptosis. Immunity 1996; 5: 449-460.

17. Buzzai M, Jones RG, Amaravadi RK, Lum JJ, DeBerardinis RJ, Zhao FP et al. Systemic treatment with the antidiabetic drug metformin selectively impairs p53-deficient tumor cell growth. Cancer Res 2007; 67: 6745-6752.

18. Munoz-Pinedo C, El Mjiyad N, Ricci JE. Cancer metabolism: current perspectives and future directions. Cell Death Dis 2012; 3: e248.

19. Deng XS, Wang S, Deng A, Liu B, Edgerton SM, Lind SE et al. Metformin targets Stat3 to inhibit cell growth and induce apoptosis in triple-negative breast cancers. Cell Cycle 2012; 11: $367-376$.

20. Tomic T, Botton T, Cerezo M, Robert G, Luciano F, Puissant A et al. Metformin inhibits melanoma development through autophagy and apoptosis mechanisms. Cell Death Disease 2011; 2: e199.

21. Pattingre S, Tassa A, Qu X, Garuti R, Liang XH, Mizushima N et al. Bcl-2 antiapoptotic proteins inhibit Beclin 1-dependent autophagy. Cell 2005; 122: 927-939.

22. Komatsu M, Waguri S, Koike M, Sou YS, Ueno T, Hara T et al. Homeostatic levels of p62 control cytoplasmic inclusion body formation in autophagy-deficient mice. Cell 2007; 131: 1149-1163.

23. Sun $K$, Guo XL, Zhao QD, Jing YY, Kou XR, Xie XQ et al. Paradoxical role of autophagy in the dysplastic and tumor-forming stages of hepatocarcinoma development in rats. Cell Death Disease 2013; 4: e501.

24. Kimmelman AC. The dynamic nature of autophagy in cancer. Genes Dev 2011; 25: 1999-2010.

25. White DE, Kurpios NA, Zuo D, Hassell JA, Blaess S, Mueller U et al. Targeted disruption of beta1-integrin in a transgenic mouse model of human breast cancer reveals an essential role in mammary tumor induction. Cancer Cell 2004; 6: 159-170.

26. Lu Z, Luo RZ, Lu Y, Zhang X, Yu Q, Khare S et al. The tumor suppressor gene ARHI regulates autophagy and tumor dormancy in human ovarian cancer cells. $J$ Clin Invest 2008; 118: 3917-3929.

27. Marino G, Ugalde AP, Salvador-Montoliu N, Varela I, Quiros PM, Cadinanos J et al. Premature aging in mice activates a systemic metabolic response involving autophagy induction. Hum Mol Genet 2008; 17: 2196-2211.

28. Akar U, Chaves-Reyez A, Barria M, Tari A, Sanguino A, Kondo Y et al. Silencing of Bcl-2 expression by small interfering RNA induces autophagic cell death in MCF-7 breast cancer cells. Autophagy 2008; 4: 669-679. 
29. Shimizu S, Kanaseki T, Mizushima N, Mizuta T, Arakawa-Kobayashi S, Thompson CB et al. Role of $\mathrm{Bcl}-2$ family proteins in a non-apoptotic programmed cell death dependent on autophagy genes. Nat Cell Biol 2004; 6: 1221-1228.

30. Pattingre S, Levine B. Bcl-2 inhibition of autophagy: a new route to cancer? Cancer Res 2006; 66: 2885-2888.

31. Shi WY, Xiao D, Wang L, Dong LH, Yan ZX, Shen ZX et al. Therapeutic metformin/AMPK activation blocked lymphoma cell growth via inhibition of mTOR pathway and induction of autophagy. Cell Death Dis 2012; 3: e275.

32. Zakikhani M, Dowling R, Fantus IG, Sonenberg N, Pollak M. Metformin is an AMP kinase-dependent growth inhibitor for breast cancer cells. Cancer Res 2006; 66: 10269-10273.

33. Skinner HD, McCurdy MR, Echeverria AE, Lin SH, Welsh JW, O'Reilly MS et al. Metformin use and improved response to therapy in esophageal adenocarcinoma. Acta Oncol 2013; 52: $1002-1009$.

34. Kobayashi M, Kato K, Iwama H, Fujihara S, Nishiyama N, Mimura S et al. Antitumor effect of metformin in esophageal cancer: in vitro study. Int J Oncol 2013; 42: 517-524.

35. Maiuri MC, Zalckvar E, Kimchi A, Kroemer G. Self-eating and self-killing: crosstalk between autophagy and apoptosis. Nat Rev Mol Cell Biol 2007; 8: 741-752.

36. Ben Sahra I, Laurent K, Giuliano S, Larbret F, Ponzio G, Gounon P et al. Targeting cancer cell metabolism: the combination of metformin and 2-deoxyglucose induces p53-dependent apoptosis in prostate cancer cells. Cancer Res 2010; 70: 2465-2475.

37. Hsia JY, Chen CY, Hsu CP, Shai SE, Yang SS, Chuang CY et al. Expression of apoptosisregulating proteins $\mathrm{p} 53, \mathrm{Bcl}-2$, and $\mathrm{Bax}$ in primary resected esophageal squamous cell carcinoma. Neoplasma 2001; 48: 483-488.

38. Yokoyama T, Kondo $\mathrm{Y}$, Kondo $\mathrm{S}$. Roles of mTOR and STAT3 in autophagy induced by telomere 3' overhang-specific DNA oligonucleotides. Autophagy 2007; 3: 496-498.

39. Yoon S, Woo SU, Kang JH, Kim K, Kwon MH, Park S et al. STAT3 transcriptional factor activated by reactive oxygen species induces IL 6 in starvation-induced autophagy of cancer cells. Autophagy 2010; 6: 1125-1138.
40. Elschami M, Scherr M, Philippens B, Gerardy-Schahn R. Reduction of STAT3 expression induces mitochondrial dysfunction and autophagy in cardiac HL-1 cells. Eur J Cell Biol 2013; 92: 21-29.

41. Nielsen M, Kaestel CG, Eriksen KW, Woetmann A, Stokkedal T, Kaltoft K et al. Inhibition of constitutively activated Stat3 correlates with altered Bcl-2/Bax expression and induction of apoptosis in mycosis fungoides tumor cells. Leukemia 1999; 13: 735-738.

42. Zhang H, Jin Y, Chen X, Jin C, Law S, Tsao SW et al. Cytogenetic aberrations in immortalization of esophageal epithelial cells. Cancer Genet Cytogenet 2006; 165 25-35.

43. Dong H, Guo H, Xie L, Wang G, Zhong X, Khoury T et al. The metastasis-associated gene MTA3, a component of the Mi-2/NuRD transcriptional repression complex, predicts prognosis of gastroesophageal junction adenocarcinoma. PLoS One 2013; 8: e62986.

44. Li Z, Zou X, Xie L, Dong H, Chen Y, Liu Q et al. Prognostic importance and therapeutic implications of PAK1, a drugable protein kinase, in gastroesophageal junction adenocarcinoma. PLoS One 2013; 8: e80665.

45. Zhang H, Kuang SQ, Liao L, Zhou S, Xu J. Haploid inactivation of the amplified-in-breast cancer 3 coactivator reduces the inhibitory effect of peroxisome proliferator-activated receptor gamma and retinoid $\mathrm{X}$ receptor on cell proliferation and accelerates polyoma middle-T antigen-induced mammary tumorigenesis in mice. Cancer Res 2004; 64: 7169-7177.

46. Tasdemir E, Galluzzi L, Maiuri MC, Criollo A, Vitale I, Hangen E et al. Methods for assessing autophagy and autophagic cell death. Methods Mol Biol 2008; 445: 29-76.

(i) () $\Theta$ Cell Death and Disease is an open-access journal published by Nature Publishing Group. This work is licensed under a Creative Commons Attribution-NonCommercialNoDerivs 3.0 Unported License. To view a copy of this license, visit http://creativecommons.org/licenses/by-nc-nd/3.0/

Supplementary Information accompanies this paper on Cell Death and Disease website (http://www.nature.com/cddis) 